\title{
The Large Binocular Telescope Interferometer \& Adaptive Optics System: On-sky Performance and Results
}

\author{
Vanessa Bailey $^{1}$, Philip Hinz ${ }^{1}$, Vidhya Vaitheeswaran ${ }^{1}$, Andrew \\ Skemer $^{1}$, Denis Defrère ${ }^{1}$, Timothy Rodigas ${ }^{1}$, Simone Esposito ${ }^{2}$, \\ Enrico Pinna ${ }^{2}$ and Alfio Puglisi ${ }^{2}$ \\ ${ }^{1}$ Steward Observatory, University of Arizona, 933 N Cherry Ave., Tucson AZ, 85719 \\ ${ }^{2}$ Istituto Nazionale di Astrofisica, Osservatorio Astrofisico di Arcetri, \\ Largo Enrico Fermi 5, I - 50125, Florence Italy \\ email: vbailey@as.arizona.edu
}

\begin{abstract}
Increasing spatial resolution and contrast capabilities will make possible new direct detections of exoplanets, exozodis, and circumstellar disks. The Large Binocular Telescope Interferometer (LBTI) has been engineered to sit at the combined focus of the Large Binocular Telescope's two $8.4 \mathrm{~m}$ apertures. Both apertures are equipped with 672-actuator deformable secondary mirrors, the first of the next generation of "extreme" adaptive optics (AO) systems. We present an overview of the LBTI AO instrument suite and detail current on-sky performance.
\end{abstract}

Keywords. instrumentation: adaptive optics, instrumentation: interferometers, instrumentation: high angular resolution

Direct imaging of exoplanets and circumstellar disks delivers exciting scientific results, but requires high-quality, stable Point Spread Functions (PSFs). Direct imaging allows for detailed study of the atmospheric properties of planets akin to gas giants in our own outer solar system (e.g.: Konopacky et al. 2013). In order to lay the framework for future imaging searches for exo-Earths, we will first need to understand the properties of exozodis, dust belts akin to our own zodical dust (e.g.: Millan-Gabet et al. 2011). In each case we are limited by the planet-to-star contrast ratio and angular resolution that can be delivered by the current generation of adaptive optics and imaging systems.

In light of these requirements, the Large Binocular Telescope Interferometer (LBTI) has been designed to sit at the combined focus of the Large Binocular Telescope's (LBT) two $8.4 \mathrm{~m}$, adaptive optics-fed apertures. Each aperture independently delivers stable, diffraction-limited images, ideal for exoplanet and cicumstellar disk searches at $3-4 \mu \mathrm{m}$. When the beams are combined interferometrically, the $23 \mathrm{~m}$ baseline between the apertures allows LBTI to resolve faint exozodi emission around nearby stars at $10 \mu \mathrm{m}$. Both of these operational modes are made possible by LBTI's dual, high-contrast adaptive optics (AO) systems.

The LBTI natural guide star AO system is one of only two AO systems in the world which use adaptive secondary mirrors (ASMs) and pyramid wavefront sensors (PWFSs; Ragazzoni 1996), the other being the Magellan Clay telescope (Close et al. 2012). LBTI's PWFSs are clones of the design used in the LBT FLAO system (Esposito et al. 2011). The two LBT ASMs themselves are $0.9 \mathrm{~m}$ in diameter, each with 672 actuators. The high actuator density allows for correction of up to 400 aberration modes. By replacing a fixed secondary with a deformable one, no additional elements are added to the optical path. This design is particularly beneficial at wavelengths $>3 \mu \mathrm{m}$, where each warm optic 

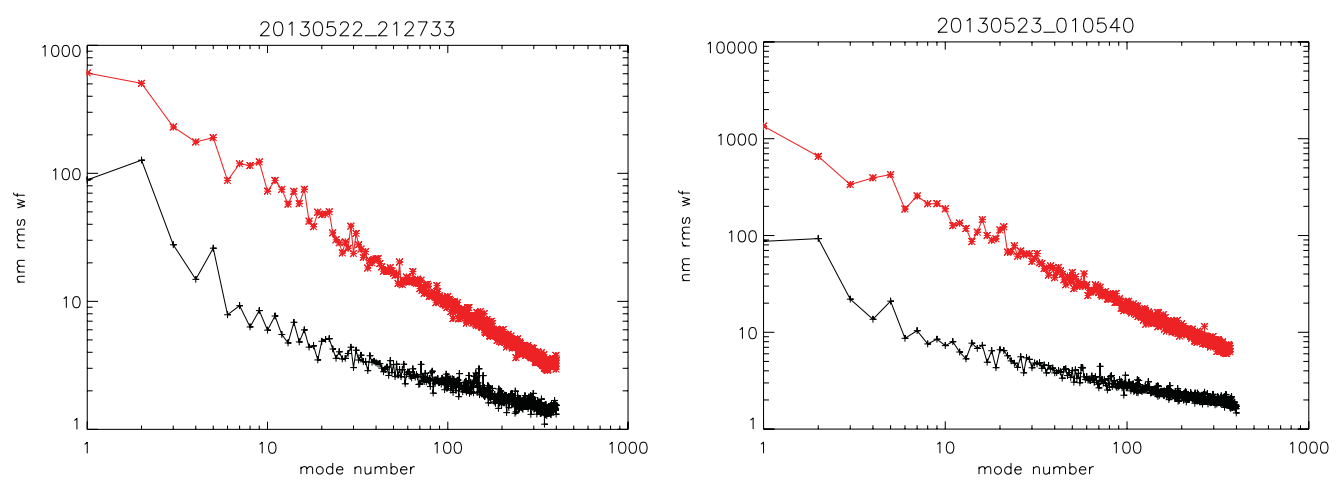

Figure 1. LBTI AO performance (RMS wavefront error) as a function of mode number. Low mode numbers correspond to low spatial frequencies (e.g.: tip, tilt, focus, astigmatism...), while high mode numbers correspond to high spatial frequencies. The upper line $(-*-)$ is the atmospheric power spectrum, while the lower line (-+-) is the power spectrum of the corrected wavefront. Left: Natural seeing $\sim 0.8^{\prime \prime}$. Right: Natural seeing $\sim 1.3^{\prime \prime}$.

contributes to the overall background flux, decreasing the science camera sensitivity (Lloyd-Hart et al. 2000). Another benefit to using an ASM on a Gregorian telescope is the ability to calibrate the AO system after it has been installed on the telescope. By calibrating in-situ, the the mirror actuators and wavefront sensor may be precisionaligned and their on-sky configuration duplicated.

The PWFS was chosen because it provides improved performance and flexibility as compared to traditional Shack-Hartmann sensors. One especially advantageous aspect of PWFS, compared to Shack-Hartmann sensors, is the ability to bin the WFS CCD for improved sensitivity on faint stars. The LBTI AO system can guide on stars as faint as $R=15$, with an ultimate goal of $R=16$ or fainter, for improved sky coverage.

Excellent on-sky performance has been demonstrated in the last year, with up to 400 spatial aberration modes corrected. Figure 1 shows the improvement in wavefront error for bright targets $(R=5-6)$ as measured by the AO system. We achieve a factor of 5 improvement in cumulative wavefront error in good seeing (0.8"), and a factor of 9 in moderate seeing (1.3"). The greatest net gains are made at low modes (like tip/tilt, astigmatism, etc.), though even at the highest spatial frequencies, the system still reduces the RMS error by a factor of $3-7$.

The quality and stability of the resulting PSF have enabled exceptional high-contrast imaging results and will be the basis for the next generation nulling interferometery survey. LBTI has already achieved an $L^{\prime}$ contrast of 13 magnitudes at 0.75 " and its nulling interferometry channel will ultimately reach sensitivities of 10 zodis (see companion papers by D. Defrere et al., T. Rodigas et al., and A. Skemer et al.).

\section{References}

Close, L., et al. 2012, SPIE, 8447, 84470X

Esposito, S., et al. 2011, SPIE, 8149, 814902

Lloyd-Hart, M. 2000, PASP, 768, 264

Konopacky, Q., et al. 2013, Science, 339, 1398

Millan-Gabet, R., et al. 2011, ApJ, 734, 67

Ragazzoni, R. 1996, JModOpt, 43, 289

Lloyd-Hart, M. 2000, PASP, 112, 264 